\title{
STABILITY AGAINST LOCALIZATION OF SOFTENING INTO ELLIPSOIDS AND BANDS: PARAMETER STUDY
}

\author{
ZDENĚK P. BAŽANT \\ Department of Civil Engineering. Northwestern University, Evanston, IL 60208, U.S.A. \\ and \\ FENG-Bao LiN \\ Department of Civil and Environmental Engineering. Polytechnic University. Brooklyn, NY 11201 , \\ U.S.A.
}

(Received 10 October 1987: in revised form 29 November 1988)

\begin{abstract}
Strain-localization instabilities due to strain-softening which result from distributed damage such as cracking in heterogeneous brittle materials are analysed. Attention is restricted to the stability problem of equilibrium states. This problem is not equivalent to bifurcation of the equilibrium path. which may occur before stability of equilibrium is lost. The continuum is local but is enhanced by the loxalization limiter used in the crack band model, consisting of a lower bound on the minimum dimension of the strain-localization region. which is regarded as a material property. Presented are derivations of the critical state conditions for localization of initially uniform strain into ellipsoidal domains within an infinite continuum and into a planar hand within a layer of tinite thickness. These derivations are simpler than the previous Bazzant's derivations of the general stability conditions for these localizations. A numerical parameter study of the critical states is made for a broad range of material propertics as well as various initial stress states and relative sines of the strain-softening region. The material is described by Drucker Prager plasticity with strain-softening that is caused hy yiedd limit degradation. The flatter the ellipsoidal domain, or the larger the size of the bedy (layer thickness), the snlaller is found to be the strain-softening slope magnilude at which the critical state is reilched. A softening Drucker Prager material is found to be stable against planar-band localizations in inlinite continuum for a certain range of softening malcrial paramelers.
\end{abstract}

\section{INTRODUCTION}

Distributed damatge such as cracking or void growth can be macroscopically described as strain-softening, a behavior in which the stress declines at increasing strain, or more precisely the matrix of incremental elastic moduli ceases to be positive definite. Strain-softening catuses the strain as well as the energy dissipation to localize. This localization represents a stability problem.

For one-dimensional localization that describes the tensile or compressive failure of a uniaxially stressed bar as well as the development of a planar localization band (e.g. crack band) in infinite space, the stability conditions in terms of the strain-softening properties of the material were derived in a 1974 report and a follow-up paper by Bažant (1976). From this stability analysis it transpired that, in the usual local continuum, the strain as well as the energy dissipation localize into a region of zero volume. This implies the structure to fail with a zero energy dissipation and the failure to occur right after the first onset of localization. This would indicate every strain-softening state to be unstable and therefore unobservable. In reality, structures of course fail with a finite energy dissipation and strainsoftening states in which the energy dissipation is not localized to a zero volume do exist, as evidenced e.g. by measurements of the locations of sound emission sources.

The simplest remedy that eliminates this physically unrealistic situation is to impose a lower bound on the minimum cross-section dimension of the strain-softening region. This ad hoc measure was introduced by Bazant (1976) in the finite element crack band model, and was subsequently developed first for sudden softening (Bažant and Cedolin, 1979, 1980) and later for gradual softening (Bažant. 1982; Bažant and Oh. 1983). The last version has been shown to be in good agreement with all the basic concrete fracture data, and to exhibit the correct size effect. which is transitional between the size effect of plastic limit analysis and the size effect of linear elastic fracture mechanics. The crack band model has 
further been refined and implemented in some large finite element codes (de Borst, 1984; de Borst and Nauta, 1984, 1985 : Darwin. 1985; Bažant. 1986). It will therefore be assumed in this study that strain-softening cannot localize into a region whose minimum crosssection dimension is less than a certain characteristic length $h$, which is considered to be a material property.

The strain localization instability was explained and analyzed in 1974 by Bažant (1976) for one-dimensional strain-softening in bars of infinite or finite length with rigid or elastic supports, as well as for flexural softening in beams: see also Bažant’s (1986) review. Rudnicki and Rice (1975) formulated the condition of localization into a planar band in an infinite space (see also Rice. 1976). This condition turned out to be identical to the conditions of uniqueness and of shear band formation, previously obtained by Hill (1962) (see also Mandel. 1966 and Mróz, 1966). The study of Rudnicki and Rice (1975) was focused primarily on localization instabilities caused by geometrically nonlinear effects of strain. which they showed to be possible already before the peak of the stress-strain diagram (i.e. in the plastic-hardening range). However, some critical states were also obtained for negative values of the plastic-hardening modulus, i.e. in the softening range. These studies, which were restricted to nonassociated Drucker-Prager plasticity, in some cases enhanced with a vertex hardening term, did not consider bodies of finite dimensions for which the size of the localization region usually has paramount influence on the critical state, and did not generally treat unloading outside the localization band, which is essential for the loss of stability in finite bodies. Subsequently, Rudnicki (1977) studied localization into ellipsoidal regions in a uniformly stressed infinite space and showed examples of such instabilities in the hardening as well as softening regime. This study was also limited to nonassociated Drucker-Prager plasticity (without or with a vertex hardening term), and dealt only with the critical state of neutral equilibrium while stability was not analyzed.

Recently Bažant (1988:a, b) formulated in a closed form the conditions of critical state as well as stability for the localization of strain-softening into planar bands or ellipsoidal regions. This study included formulation of the stability conditions for strain-softening localization into a planar band that forms within a layer of finite thickness. These stability conditions allowed for completely general material properties characterized by arbitrary tensors of incremental moduli for loading and unloading (with general anisotropy), which makes it possible to determine the important effect of the type of constitutive law on these instabilities. The numerical examples, however, dealt only with the special case of isotropic incremental elastic moduli tensors, which is simple to treat but of course not too realistic.

Our objective will be to apply these previously derived stability conditions assuming more real listic, incrementally anisotropic material properties based on Drucker-Prager plaslicity (Fung, 1965; Owen and Hinton, 1980; Chen, 1982). At the same time, we will present a direct derivation of the conditions of critical state (stability limit) which is considerably simpler than the previous derivation of the stability conditions. Compared to the work of Rudnicki and Rice (1975) and Rice (1976), the present study as well as the preceding ones by Batzant $(1988 \mathrm{a}, \mathrm{b})$ extends the localization condition to arbitrary constitutive laws and to bodies of finite dimensions for which unloading is important and the body size matters.

It needs to be emphasized that, as in Bažant's (1988a, b) preceding study, the present study deals only with the critical state of the loss of stability of equilibrium, and not with bifurcation of the equilibrium path as the load is increased. The problems of stability loss and bifurcation are not equivalent. For uniaxially stressed bars or planar localization bands, the critical state for stability differs from the state of bifurcation (Bažant, 1988c). More detailed comments on this subject will be made later in this paper.

To a void localization of energy dissipation into a region of zero volume, one generally needs to introduce the so-called localization limiters (Bažant and Belytschko, 1987). The imposition of a lower bound on the size of this region, adopted in this study, is the simplest but crudest localization limiter. Limitation of localization in general calls for adopting the nonlocal continuum approach (Bažant et al., 1984; Bažant, 1987). The latest form of this approach. which is easily implemented in large finite element codes, is the nonlocal continuum with local strain (Bažant and Pijaudier-Cabot. 1987. 1988; Pijaudier-Cabot and Bažant. 1987: Bažant and Lin, 1988). The nonlocal approach, however, does not seem 


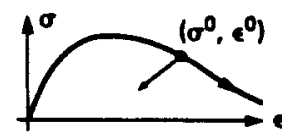

(a)

(b) (c)

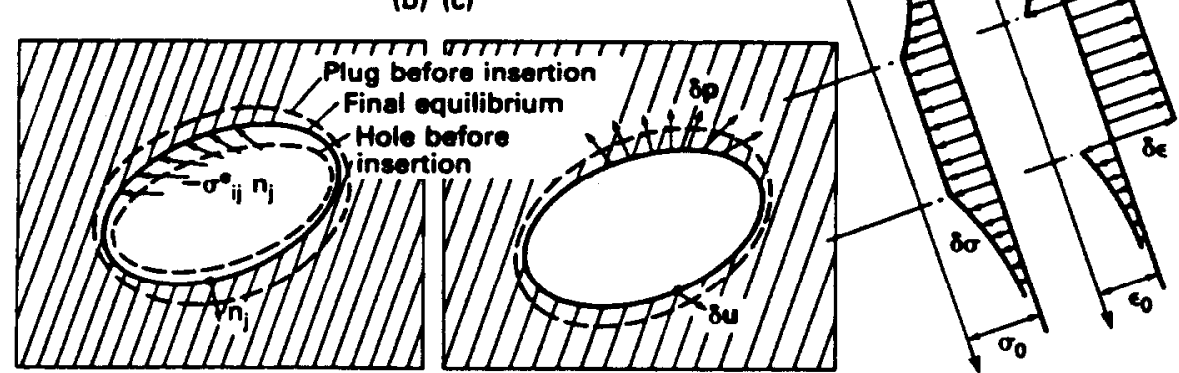

Fig. I. Ellipsoidal plug (inclusion) inserted into infinite elastic solid, and localization of strain into an elliptic region.

amenable to closed-form expressions for the stability conditions and therefore will not be pursued here.

Compared to the general nonlocal formulation, the use of a local continuum with the simple imposition of a lower limit on the size of the localization zone, made in the crack band model and adopted here, might seem too simplistic. The numerical results obtained with this model have nevertheless been found to be rather close to the nonlocal solutions (Bažant and Pijaudier-Cabot, 1988; Pijaudier-Cabot and Bažant, 1987). This is a supporting argument for the present approach.

\section{CRITICAL STATE CONDITIONS FOR LOCALIZATION INTO ELLIPSOIDS}

First we consider an ellipsoidal hole (Fig. Ib) in an infinite homogeneous elastic continuum characterized by the elastic moduli matrix $D_{u}$. We imagine to fit and glue into this hole an ellipsoidal plug made of the same material. To fit, the plug must first be deformed by a uniform strain, $\varepsilon^{\circ}$, called the eigenstrain (note that an ellipsoid can be transformed to any other ellipsoid by uniform strain). The eigenstrain is then imagined unfrozen, which causes the plug with ellipsoidal hole to undergo strain increment $e^{e}$ in order to establish equilibrium with the surrounding infinite continuum. According to the celebrated Eshelby's theorem (Eshelby, 1957; Christensen, 1979; Mura, 1982), the strain $e^{c}$ in the plug is uniform and is expressed as

$$
\varepsilon_{i j}^{e}=S_{i j k m} \varepsilon_{k m}^{0}
$$

in which $S_{i j k m}$ are the components of a fourth-rank tensor which depend only on the aspect ratios $a_{1} / a_{2}, a_{3} / a_{2}$ of the principal axes of the ellipsoid, as well as on the elastic moduli. Eshelby's coefficients $S_{i / k m}$ are generally calculated as elliptic integrals (Mura, 1982). Always, $S_{i j k m}=S_{j i k m}=S_{i j m k}$, but in general $S_{i j k m} \neq S_{k m t j}$. For generally anisotropic material properties, the expressions for coefficients $S_{k m u}$ were derived by Kinoshita and Mura (1971) and Lin and Mura (1973).

For convenience we rewrite eqn (1) in a matrix form:

$$
\left\{\begin{array}{c}
\varepsilon_{11}^{c} \\
\varepsilon_{22}^{c} \\
\varepsilon_{33}^{c} \\
2 \varepsilon_{12}^{c} \\
2 \varepsilon_{23}^{c} \\
2 \varepsilon_{31}^{c}
\end{array}\right\}=\left[\begin{array}{ccc:ccc}
S_{1111} & S_{1122} & S_{1133} & S_{1112} & S_{1123} & S_{1131} \\
S_{2211} & S_{2222} & S_{2233} & S_{2212} & S_{2223} & S_{2231} \\
S_{3311} & S_{3322} & S_{333} & S_{3312} & S_{3323} & S_{3331} \\
\hdashline 2 S_{1211} & 2 S_{1222} & 2 S_{1233} & 2 S_{1212} & 2 S_{1223} & 2 S_{1231} \\
2 S_{2311} & 2 S_{2322} & 2 S_{2333} & 2 S_{2312} & 2 S_{2323} & 2 S_{2331} \\
2 S_{3111} & 2 S_{3122} & 2 S_{3133} & 2 S_{3112} & 2 S_{3123} & 2 S_{3131}
\end{array}\right]\left\{\begin{array}{c}
\varepsilon_{11}^{0} \\
\varepsilon_{22}^{0} \\
\varepsilon_{33}^{0} \\
2 \varepsilon_{12}^{0} \\
2 \varepsilon_{23}^{0} \\
2 \varepsilon_{31}^{0}
\end{array}\right\}
$$


or

$$
\varepsilon^{\varepsilon}=Q_{u} \varepsilon^{0}
$$

in which $\varepsilon^{0}$ and $\varepsilon^{e}$ are the column matrices of the eigenstrains and the equilibrium strains in the ellipsoidal region. These column matrices (with the factors 2 ) are defined in such a manner that $\sigma^{\mathrm{T}} \varepsilon$ is the correct expression for work density $; \sigma=\left(\sigma_{11}, \sigma_{22}, \sigma_{33}, \sigma_{12}, \sigma_{23}\right.$, $\left.\sigma_{31}\right)^{\mathrm{T}}=$ column matrix of stresses (superscript $\mathrm{T}$ denotes the transpose of a matrix). For isotropic materials, the only nonzero elements of matrix $Q$ of Eshelby's coefficients are those between the dashed lines marked in eqn (2).

According to Hooke's law, the stress in the ellipsoidal plug, $\sigma^{e}$, which is uniform, may be expressed as $\sigma^{e}=D_{u}\left(\varepsilon^{e}-\varepsilon^{0}\right)$. Upon substituting $\varepsilon^{0}=Q_{u}^{-1} \varepsilon^{e}$ [according to eqn (2)], we obtain

$$
\sigma^{e}=D_{u}\left(1-Q_{u}^{-1}\right) \varepsilon^{e}
$$

in which $1=$ unit $6 \times 6$ matrix.

Consider now that an infinite continuum (without any hole) is in an initial equilibrium state of uniform initial strain $\varepsilon$ and uniform initial stress $\sigma$ balanced by loads applied at infinity. We seek the condition under which the initial state loses stability in a mode in which the strain localizes into an ellipsoidal region (Fig. 1) without changing the prescribed stresses (or the prescribed displacements) at infinity. If these variations can happen while maintaining equilibrium. we have a state of neutral equilibrium which represents the limit of stability, i.e. the critical state.

Due to localization, the strain and stress in the infinite continuum outside the ellipsoidal region become nonuniform, while according to Eshelby's theorem the stress and strain inside the ellipsoidal region will remain uniform. The strains can become discontinuous across the ellipsoidal surface, as shown in Fig. Id. On the other hand, the normal and shear stress components acting on this surface must remain continuous in order to maintain equilibrium (while the normal stresses parallel to the ellipsoidal surface need not be continuous). If the ellipsoidal region undergoes strain-softening, the outside undergoes unloading and therefore behaves elastically. According to eqn (3), the stress variations immediately outside the ellipsoidal region are $\delta \sigma^{e}=D_{u}\left(1-Q_{u}^{-1}\right) \delta \varepsilon^{e}$ in which $D_{u}$ must now be interpreted as the matrix of elastic moduli for unloading, corresponding to the initial strain 8 . This matrix is positive definite, and it is also isotropic if the material is isotropic. The uniform stress variations inside the ellipsoidal region are $\delta \sigma^{\prime}=\mathrm{D}, \delta e^{e}$, in which $\mathrm{D}$, is the matrix of incremental elastic moduli for further loading, corresponding to the initial strain $e$. This matrix is not positive definite if the initial state $\varepsilon$ is in the strain-softening range, and generally it must be assumed to be anisotropic. Equilibrium of the ellipsoidal region with its exterior is maintained if $\delta \sigma^{t}=\delta \sigma^{e}$. From this we obtain the following conditions of neutral equilibrium (i.e. critical state) :

$$
\left[D_{t}-D_{u}\left(1-Q_{u}^{-1}\right)\right] \delta \varepsilon^{e}=0 .
$$

It may be noted that for each point of ellipsoidal surface, at which the unit normal is $\mathbf{n}$, equilibrium requires only that $\delta \sigma^{\prime} \mathbf{n}=\delta \sigma^{\mathbf{c}} \mathbf{n}$. However, due to the fact that this condition must hold for various $n$, it is necessary that $\delta \sigma^{f}=\delta \sigma^{c}$.

Equation (4) represents a system of six homogeneous linear algebraic equations for the six components of $\delta \varepsilon^{e}$. A nonzero solution, which represents the strain localization instability mode, is possible if and only if the determinant of this equation system vanishes, i.e.

$$
\text { Det } Z=0 \text {, with } Z=D_{\imath}-D_{u}\left(1-Q_{u}^{-1}\right) \text {. }
$$

This is the same result as obtained previously in Bažant (1988b), in which it was further shown that the initial state of uniform strain $\varepsilon$ in unstable if matrix $\mathbf{Z}$ is not positive definite, and is stable (with regard to the presently assumed localization mode) if this matrix is positive definite. This result was obtained (Bažant, 1988b) by analyzing the sign of the 
second-order work $\delta^{2} W$ needed to produce an equilibrium localization increment. This is the work that is done by the interfacial tractions applied on the ellipsoidal surface during an equilibrium localization increment. It has been shown that $\delta^{2} W=\delta e^{\mathrm{r}} \mathrm{Z} \delta e^{\mathrm{e}} V / 2=$ $-T(\Delta S)_{\text {in }}$ where $(\Delta S)_{\text {in }}$ is the increment of internally produced entropy of the body and $V=$ volume of the ellipsoidal region (see Bażant, 1988c).

Due to internal friction, damage or other phenomena, matrix $\mathbf{D}_{l}$, and thus also $\mathbf{Z}$, can be nonsymmetric. In that case. the states for which $Z$ is $\operatorname{singular}(\operatorname{det} Z=0$ ) represent only the states of neutral equilibrium but not the states at the limit of stability (i.e. limit of positive definiteness of $\mathbf{Z}$ ). Since $2 \delta^{2} W / V=\delta \varepsilon^{\varepsilon^{\top}} Z \delta \varepsilon^{e}=\delta \varepsilon^{e^{\top}} \mathbf{Z} \delta \varepsilon^{e}$ for any $\delta \varepsilon^{e^{\top}}$, where $Z=$ $\frac{1}{2}\left(\mathbf{Z}+\mathbf{Z}^{\mathbf{T}}\right)=$ symmetric part of matrix $\mathbf{Z}$. the limit of stable states is characterized by the singularity of matrix $Z$ (rather than $Z$ ). So, in the case of nonsymmetric matrix $D_{t}$, one needs to distinguish between two kinds of critical states:

(1) the critical state of neutral equilibrium, which is characterized by singularity of matrix $Z$. i.e. eqn (5) (in which case one eigenvalue of $Z$ is zero) ;

(2) the critical state of stability limit (i.e. limit of positive definiteness of matrix $Z$ ), which is characterized by singularity of symmetric matrix $\mathbf{Z}$. i.e. $\operatorname{det} \mathbf{Z}=0$ (in which case the smallest eigenvalue of $\mathbf{Z}$ is zero).

The second condition is more stringent than the first one, as numerical calculations confirm. However. the numerical results in Figs 3-13 which follow show that. for non-

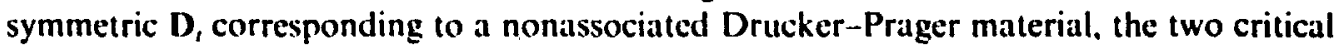
states are in most cases graphically indistinguishable. In those cases where graphical distinction is possible. the critical states of neutral equilibrium [eqn (5)] are shown as the dashed curves and the critical states of stability limit as the solid curves.

The special case of the critical state condition in eqn (5) for associated or nonassociated Drucker-Prager plasticity was previously derived by Rudnicki (1977).

\section{CRITICAL STATE CONDITIONS IOR LOCALIZATION INTO PLANAR BAND}

Consider now strain localization into an inlinite planar band of thickness $h$ that forms inside an infinite layer of thickness $L(L \geqslant h)$. We choose axis $x_{2}$ to be normal to the layer (Fig. 2). The layer is assumed to be initially in equilibrium in a state of uniform (homogeneous) strain $\varepsilon_{i j}$ and stress $\sigma_{i j}$ (the latin lower-case subscripts refer to Cartesian coordinates $\left.x_{i}, i=1,2,3\right)$. We imagine the initial equilibrium state to be disturbed by infinitesimal displacement variations $\delta u_{i}$ whose gradients $\delta u_{i, j}$ have the values $\delta u_{i, j}^{t}$ and $\delta u_{i,}^{\prime \prime}$ inside and outside the band, representing further loading or unloading. respectively. These variations are uniform within the band and also outside the band, with discontinuous jump across the surface of the band. As the boundary conditions, we consider that the surface points of the layer are fixed during the incremental deformation, i.e. $\delta u_{i}=0$ at the surfaces $x_{2}=0$ and $x_{2}=L$ of the layer. The stress variations inside the band and outside the band are:

$$
\delta \sigma_{2 i}^{\prime}=D_{2, j m}^{\prime} \delta c_{j, m}^{\prime}=D_{2, j m}^{\prime} \delta u_{j, m}^{\prime}=D_{2 i j 2}^{\prime} \delta u_{j, 2}^{\prime}
$$

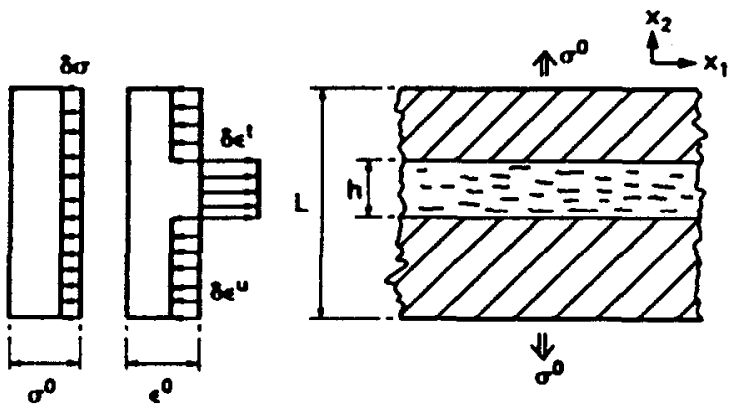

Fig. 2. Planar localization band in a layer. 


$$
\delta \sigma_{2 i}^{u}=D_{2 i j m}^{u} \delta \varepsilon_{j m}^{\mu}=D_{2 i j m}^{u} \delta u_{j, m}^{\mu}=D_{2 i, 2}^{u} \delta u_{j, 2}^{\mu}
$$

in which again $D_{k i j m}^{k}$ and $D_{k i j m}^{u}$ represent the incremental elastic moduli for further loading and for unloading. corresponding to the initial strain $\varepsilon_{i j}$. In writing eqns (6)-(7) we assume that $\delta u_{t, 1}^{u}=\delta u_{i, 1}^{i}=0, \delta u_{i, 3}^{u}=\delta u_{i, 3}^{t}=0$. Without these conditions, there would be a slip discontinuity between the inside and outside of the band.

Compatibility of deformations over the thickness of the layer requires that $h \delta u_{j, 2}^{\prime}+(L-h) \delta u_{j, 2}^{u}=0(j=1,2,3)$. Expressing $\delta u_{j, 2}^{u}$ from this equation and taking into account the condition of equilibrium at the band surfaces, $\delta \sigma_{3 i}^{u}=\delta \sigma_{3 i}^{\prime}(i=1,2,3)$, we obtain from eqns (6)-(7) the following condition of neutral equilibrium (critical state):

$$
\left(D_{2 i j 2}^{\prime}+\frac{h}{L-h} D_{2 i j 2}^{u}\right) \delta u_{j, 2}^{\prime}=0 .
$$

This represents a system of three homogeneous linear algebraic equations. A nonzero solution exists if and only if

$$
\text { Det } Z_{i j}=0, \quad \text { with } Z_{i j}=D_{2 i j 2}^{\prime}+\frac{h}{L-h} D_{2 i j 2}^{\prime}
$$

This critical state condition is again the same as obtained previously by Bažant (1988a), in which it was also proven (by analysis of the second-order work) that the states for which the matrix $Z_{i}$ is not positive definite are unstable, and the states for which it is positive definite are stable with regard to localization into a planar band. A special case of eqn (9) is the condition for uniaxial or shear localization instability derived in 1974 by Bažant (1976).

The special case of eqn (9) for nonassociated Drucker-Prager plasticity without or with vertex hardening and for infinite space (for which $L \rightarrow \infty$ and unloading $D^{*}$ plays no role) was obtained by Rudnicki and Rice (1975) and by Rudnicki (1977).

\section{BASIC PROPERTIES OF THE CRITICAL STATES}

The critical state conditions in eqns (5) and (9) were discussed in detail by Bažant $(1988 \mathrm{a}, \mathrm{b})$. Briefly, according to eqn (5) which refers to an infinite continuum, the critical state condition for localization into an ellipsoidal region is independent of the region's size, and depends only on the aspect ratios $a_{1} / a_{2}$ and $a_{3} / a_{2}$ of the ellipsoid. By contrast, according to eqn (9), which applies to a finite body, the critical state condition does depend on the size of the localization region, in this case the thickness $h$ of the band. The smaller the thickness of the band, the more stringent is the stability condition, i.e. the smaller is the magnitude of the strain-softening slope (tangent modulus) at which instability occurs. If $h \rightarrow 0$ were allowed, instability would always occur no later than at the peak stress point, and so strain-softening could never be observed. The fact that it can be observed implies that the thickness of the localization band, $h$. cannot be considered to be smaller than a certain finite length. This length must be considered to be a material property. It is roughly equal to the characteristic length / of the matcrial (Bažant and Pijaudier-Cabot, 1989).

It is now proper to comment on the difference from Hill's (1962) condition of bifurcation of the equilibrium path, $D_{2 i, 2}^{\prime} \delta u_{j, 2}^{\prime}=0$. This equation coincides with Rudnicki and Rice's (1975) condition and is obtained from eqn (8) with $L / h \rightarrow \infty$. As a bifurcation condition, however, this equation has been used [in contrast to eqn (8)] for a finite body of any size. In that case there is no unloading in the body, same as for a layer of infinite thickness. So the moduli $D_{i j k m}^{\prime}$ may be assumed to apply everywhere, which means the analysis can be made on the basis of a linear elastic body; see Hill's concept of a linear comparison solid. It must be noted, however, that the eigenmode $\delta u_{i, j}^{*}$ obtained from the equation $D_{2 i j 2}^{\prime} \delta u_{j, 2}^{\prime}=0$ violates the boundary condition of fixed displacements at the layer surface. Therefore, neutral equilibrium does not exist in the layer at the bifurcation state, and the calculated eigenmode $\delta u_{i, j}^{*}$ cannot actually occur as an instability. This means that the bifurcation must occur at increasing rather than constant boundary displacements. If 
the boundary displacement increase is controlled, such a mode of deformation does not represent stability loss, whose investigation is the objective of the present study. Rather, the bifurcation state as well as the states immediately after the bifurcation are stable.

The foregoing discussion reflects the fact that one must distinguish between the concepts of stable state and stable path, as shown in Bažant's (1988c) study. It was proven (for the special case of uniaxial stress) that after the bifurcation point. which occurs according to Hill (1962) or Rudnicki and Rice (1975) at the peak stress state. the strain must begin to localize if the material is deformed in an equilibrium manner and if the tangential moduli vary continuously as the layer is loaded in a displacement-controlled fashion. However, it was also shown that if a nonlocalized uniform post-peak state is somehow reached, this state is stable if eqn (8) is satisfied. Such a state can be reached in an equilibrium loading process if the tangential moduli decrease discontinuously during loading (or drop suddenly due to heating or other effects). Even for continuously varying moduli, such a state can be reached dynamically, or if certain temporary restraints are applied, then also statically.

According to Bażant's recent study (not yet published). the aforementioned distinction between the stability limit and the bifurcation state exists also for finite ellipsoidal regions.

The critical state conditions in eqns (5) and (9) were examined in the previous works (Bažant. 1988a.b) for the effect of material parameters. Consideration was limited to incrementally isotropic tensors $D_{i / k m}$. For the ellipsoidal localization regions, it was found that the critical slope $E_{t}^{\mathrm{ur}}$ of the stress-strain diagram $(<0)$ at which instability occurs decreases in magnitude as the ellipsoid becomes flatter, more elongated in the $x_{1}$ and $x_{3}$ directions, and becomes zero as the ellipsoid approaches an infinite band, i.e. for $a_{1} \rightarrow \infty$, $a_{3} \rightarrow \infty$. Rather large ratios $a_{1} / a_{2}$ and $a_{3} / a_{2}$, however, are needed to make $\left|E_{7}\right|$ small. For example, for $\left|E_{f}^{\mathrm{cr}}\right|$ to become less than about $5 \%$ of the unloading slope $E_{u}$. the aspect ratios $a_{1} / a_{2}$ and $a_{3} / a_{2}$ need to exceed approximately 200 . This is a surprisingly large value.

Such an clongated ellipsoidal region (whose thickness for concrete may not be less than several times the aggregate size) often cinnot be accommodated within a finite body representing a typical concrete structure. This ellipsoidal region would have to be contained within a larger region that is sufficiently remote from the surface of the body so that the boundary conditions of infinite space around the ellipsoidal region would be approximately applicable. Therefore, real structures should often be stable even for strain-softening slopes whose magnitude is much larger than that predicted by the formula for the planar band [equ (9) and also Bažant, 1976] using a characteristic length of the same order of magnitude as the maximum size of inhomogeneity in the material.

Equations (5) and (9) can be used to study the influence of material parameters on the localization instabilities for more realistic constitutive laws, for which the tensor $D_{i j k m}^{z}$ is anisotropic. We will do so now for some plastic constitutive models.

\section{PARAMETER STUDY FOR NONASSOCIATED DRUCKER-PRAGER PLASTICITY}

The plastic stress-strain relations for isotropic materials have the form:

$$
\mathrm{d} s_{i j}=2 G\left(\mathrm{~d} e_{i j}-\mathrm{d} e_{i j}^{p}\right), \quad \mathrm{d} \sigma^{n}=3 K\left(\mathrm{~d} \varepsilon^{p}-\mathrm{d} \varepsilon^{p}\right)
$$

in which $G, K=$ shear and bulk elastic moduli, $\varepsilon^{n}=\varepsilon_{k k} / 3=$ volumetric strain, $e_{i j}=\varepsilon_{i j}-$ $\delta_{i,} \varepsilon^{k}=$ deviatoric strains $\left(\delta_{i j}=\right.$ Kronecker delta), $\sigma^{\prime \prime}=\sigma_{k k} / 3=$ volumetric stress, $s_{i j}=\sigma_{i j}-$ $\delta_{i} \sigma^{*}=$ deviatoric stresses, and $\varepsilon^{p}, e_{i j}^{p}=$ volumetric and deviatoric plastic strains. The plastic strain increments are given by the flow rule:

$$
\mathrm{d} \varepsilon_{i j}^{p}=\mathrm{d} \lambda \frac{\partial g}{\partial \sigma_{i j}}
$$

in which $\mathrm{d} \lambda$ is the plastic strain parameter and $g$ is the plastic potential function. According to the plasticity theory proposed by Drucker and Prager (see Fung. 1965: Chen, 1982), the loading function and the plastic potential function are introduced as follows : 


$$
\begin{aligned}
& f(\sigma, \kappa)=\bar{\tau}+\psi\left(\sigma^{2}\right)-\kappa=0 \\
& g(\sigma, \kappa)=\bar{\tau}+\phi\left(\sigma^{n}\right)-\kappa=0
\end{aligned}
$$

in which $\bar{\tau}=J_{2}^{1}=\left(\frac{1}{2} s_{i j} s_{i j}\right)^{1 / 2}=$ stress intensity, $J_{2}=$ stress deviator, $\kappa=$ plastic hardening parameter, and $\phi, \psi=$ empirical material functions of the volumetric stress. As usual, we introduce the notations :

$$
H=\frac{\mathrm{d} \kappa}{\mathrm{d} \lambda}, \quad \beta=\frac{\partial \phi}{\partial \sigma^{n}}, \quad \beta^{\prime}=\frac{\partial \psi}{\partial \sigma^{v}},
$$

in which $H=$ plastic modulus, which is positive for hardening and negative for softening. $\beta^{\prime}=$ internal friction coefficient, and $\beta=$ dilatancy ratio. The formulation satisfies the normality rule if $g \equiv f$, which means that $\phi=\psi$ (and also $\beta=\beta^{\prime}$ ). In this case, the material is said to be associated, and otherwise nonassociated. $H, \beta$ and $\beta^{\prime \prime}$ completely characterize the incremental properties of Drucker-Prager plastic material. Traditionally $H$ has been considered positive (which describes plastic hardening, i.e. increase of the yield limit). However, allowing it to be negative provides a convenient model for strain-softening. describing degradation of the yield limit (a model of this type has been used in nonlocal analysis of tunnel cave-in; see Bažant and Lin, 1988).

On the basis of eqns (10)-(14), it can be shown by a well-known procedure that the tangential (incremental) moduli are

$$
D_{i, k m}^{\prime}=D_{i j k m}^{r}-\frac{\left(\frac{G}{\bar{t}} s_{i j}+K \beta \delta_{i j}\right)\left(\frac{G}{\bar{\tau}} s_{k m}+K \beta^{\prime} \delta_{k m}\right)}{G+K \beta \beta^{\prime}+H} .
$$

For nonassociated plasticity $\left(\beta^{\prime} \neq \beta\right)$ the tangential moduli for loading are nonsymmetric, while for associated plasticity $\left(\beta^{\prime}=\beta\right)$ they are symmetric (with respect to interchanging ij with $\mathrm{km}$ ). The tangential moduli for unloading are assumed to be the same as for initial clastic loading, and so

$$
D_{i j k m}^{u}=(K-j G) \delta_{i j} \delta_{k m}+2 G \delta_{i k} \delta_{j m} .
$$

Although the material is assumed to be isotropic. the tensor of incremental moduli for further loading [eqn (15)] exhibits stress-induced anisotropy.

After implementing the Drucker-Prager model in the computer program, it has first been checked whether the results for the case $a_{1} / a_{2} \rightarrow \infty, a_{3} / a_{2} \rightarrow \infty$ agree with the solution of Rudnicki and Rice (1975). They do.

The critical state condition in eqn (5) or (9) depends on the following four nondimensional material parameters

$$
v, H / G, \beta, \beta^{\prime}
$$

in which $v=$ Poisson's ratio. In addition it depends on $s_{i j} / \bar{\tau}$, i.e. the ratios of the initial deviatoric stresses, and also on nondimensional geometric parameters such as $L / h$ for localization in a band, and $a_{1} / a_{2}, a_{3} / a_{2}$ for localization in an ellipsoid. It does not depend on the elastic modulus $E$, however, because the division of eqn (5) or (9) by $E$ has no effect on its eigenvalues.

The effect of the aforementioned parameters has been studied numerically. The eigenvalues of matrix $Z$ in eqn (5) or matrix $Z_{i j}$ in eqn (9) have been calculated on a computer for various combinations of the parameters in eqn (17). Using Newton's iterative method, material parameter combinations for which the smallest eigenvalue vanishes have been found, and their plots are shown in Figs 3-13. The initial stress states considered in these diagrams were: uniaxial compression, biaxial compression, pure shear $\left(s_{12} \neq 0\right.$, other $s_{i j}=0$ ), and uniaxial tension. Poisson's ratio has been considered as $v=0.18$ for all the calculations. As mentioned below eqn (5), for $\beta^{\prime} \neq \beta$ there is a difference between the critical state of neutral equilibrium [det $Z=0$, eqn (5)] and the critical state of stability limit (for which the symmetric part of matrix $\mathbf{Z}$ is singular). Wherever graphically distinguishable, the former are shown as the dashed curves and the latter as the solid curves. 

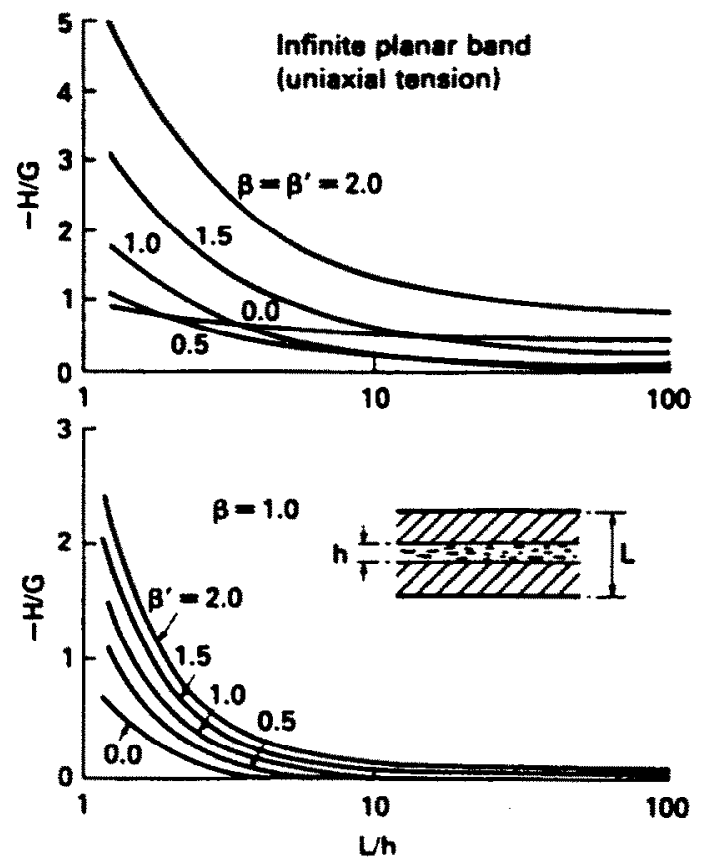

Fig. 3. Effect of size $L / h$ on critical plastic hardening modulus $H$ for infinite planar band in uniaxial tension.

Figures 3-5 show the effect of the ratio of the layer thickness $L$ to the band thickness $h$ on the value of the plastic modulus $H$ at which the stability limit is reached. The states below each curve are stable, the states above it are unstable, and the states on the curve are critical. In Figs 3-5 on top we see the curves for various values of $\beta^{\prime}$ when $\beta=\beta^{\prime}$ (i.e. for associated plasticity, for which the normality rulc is satisfied). In Figs 3-5 at the bottom we see the curves for various values of the internal friction coefficient $\beta^{\prime}$ when the dilatancy ratio is fixed as $\beta=1$. As might have been expected, the thicker the given layer, the lower is the magnitude of the plastic modulus $H$ at which the layer becomes unstable.
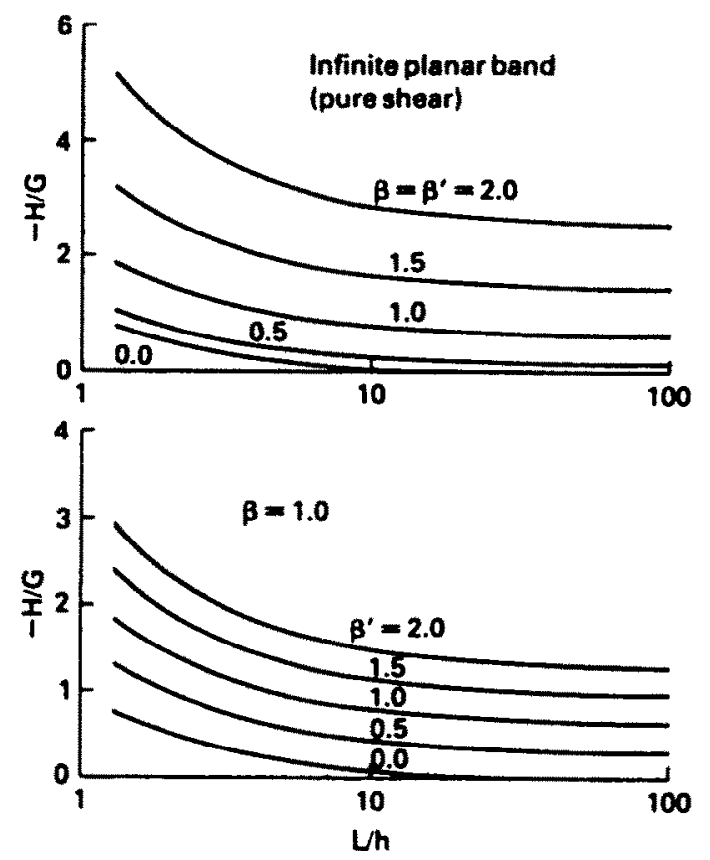

Fig. 4. Effect of size on critical $H$ for infinite planar band in pure shear. 

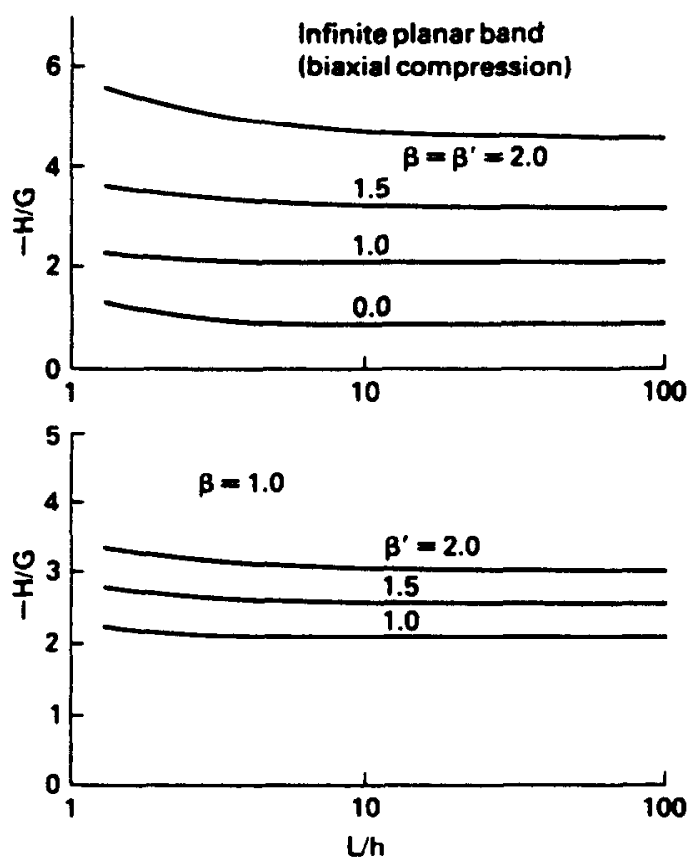

Fig. 5. Effect of size on critical $\boldsymbol{H}$ for infinite planar band in biaxial compression.

To check the limit case $L / h \rightarrow 1$, we rewrite eqn (8) as $\left[D_{2 j j 2}^{u}+D_{2 i j 2}^{\prime}(L-h) / h\right] \delta u_{j .2}^{\prime}=$ 0 . Since the equation $D_{3 i j 2}^{u} \delta u_{j, 2}^{\prime}=0$ has no nonzero solution $\left(D_{3_{i j 2}}^{u}\right.$ is positive-definite), we conclude that at least some component of $D_{21 / 2}^{\prime}$ must tend to $\infty$ to have the posssibility of localization (nonzero $\delta u_{j, 2}^{\prime}$ ). According to eqn (15) this occurs if and only if $H \rightarrow-\infty$. Therefore, the curves in Figs 3-5 tend to $\infty$ as $L / h \rightarrow 1$.

Another check on the present general numerical solution is provided by the special case of a band in an infinite space solved by Rudnicki and Rice (1975), which corresponds to $L / h \rightarrow \infty$. Indeed, their eqn (16) yields numerically the same results as the present solution for $L / h \rightarrow \infty$ and the Drucker-Prager material. It should be noted that Rudnicki and
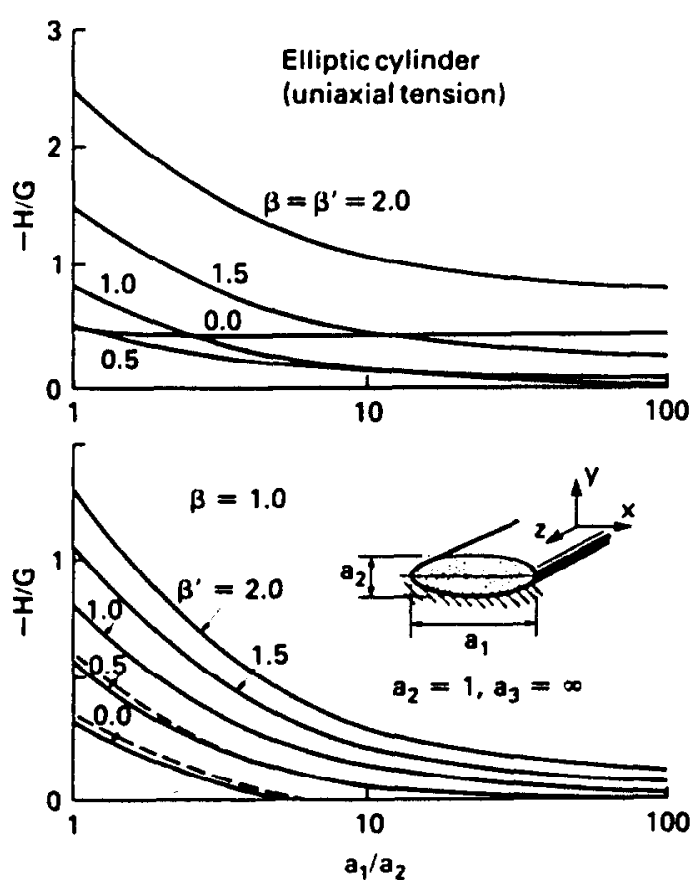

Fig. 6. Effect of ratio $a_{1} / a_{2}$ of ellipsoidal axes on critical $\boldsymbol{H}$ for elliptic cylinders in uniaxial tension. 

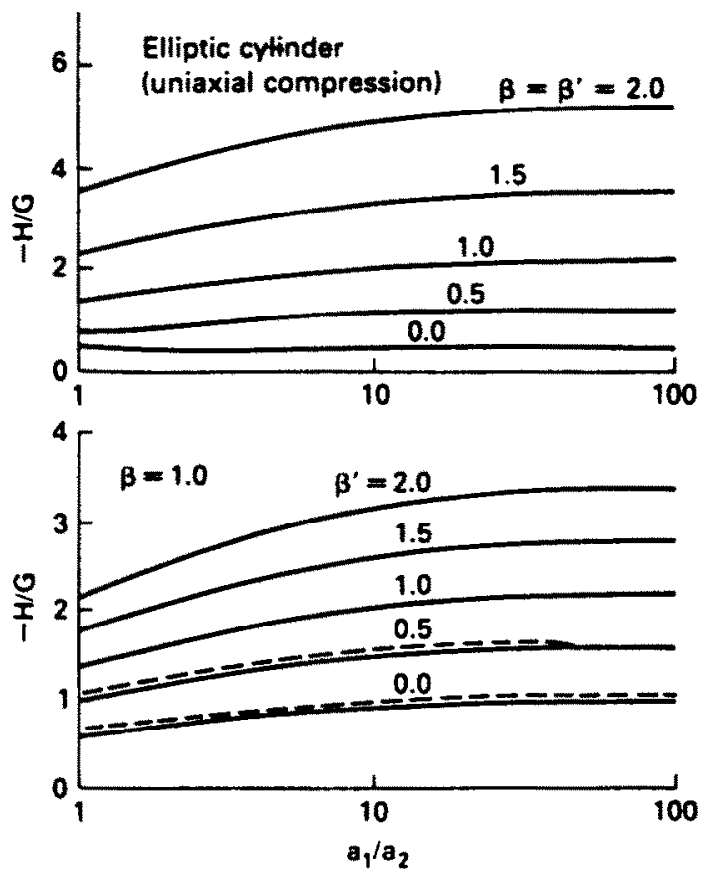

Fig. 7. Effect of axis ratio on critical $\boldsymbol{H}$ for elliptic cylinders in uniaxial compression.

Rice considered the band to have arbitrary orientation and used among the critical state conditions for all possible orientations of axis $x_{2}$ the one which is most severe. In the present study, on the other hand, the orientation of the band (axis $x_{2}$ ) is fixed because the band of thickness $h$ must be parallel to the surfaces of the layer of given thickness $L$ (except when $L \rightarrow \infty)$.

The asymptotic values of the curves are interesting. For an infinitely thick layer (i.e. infinite space), the strain localization instability does not occur at zero plastic modulus $(H \rightarrow 0)$, as might have been expected, but at a finite negative value of the plastic modulus.
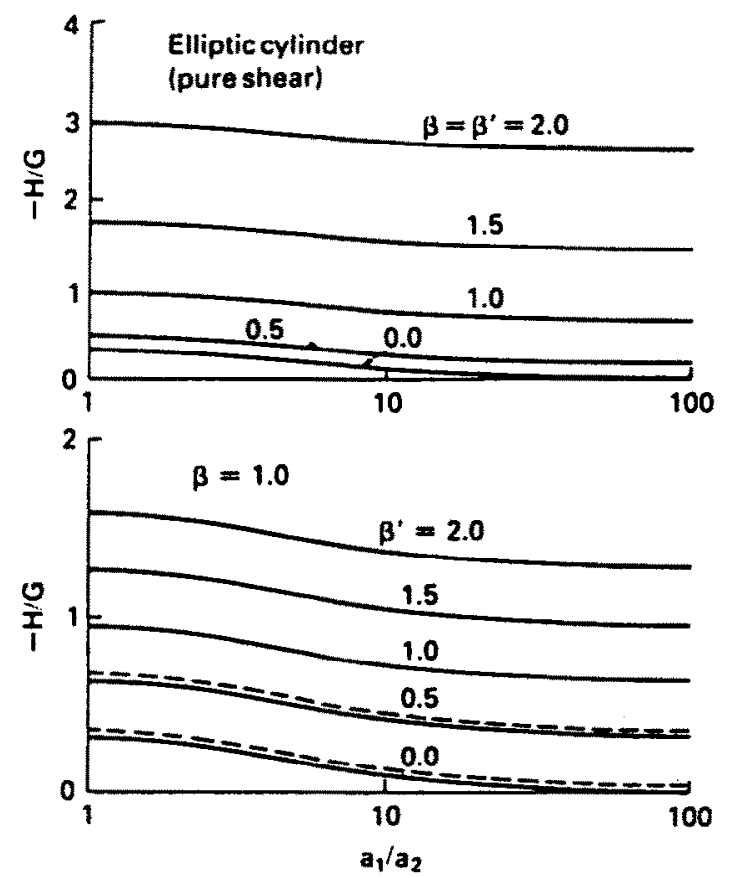

Fig. 8. Effect of axis ratio on critical $\boldsymbol{H}$ for elliptic cylinders in pure shear. 

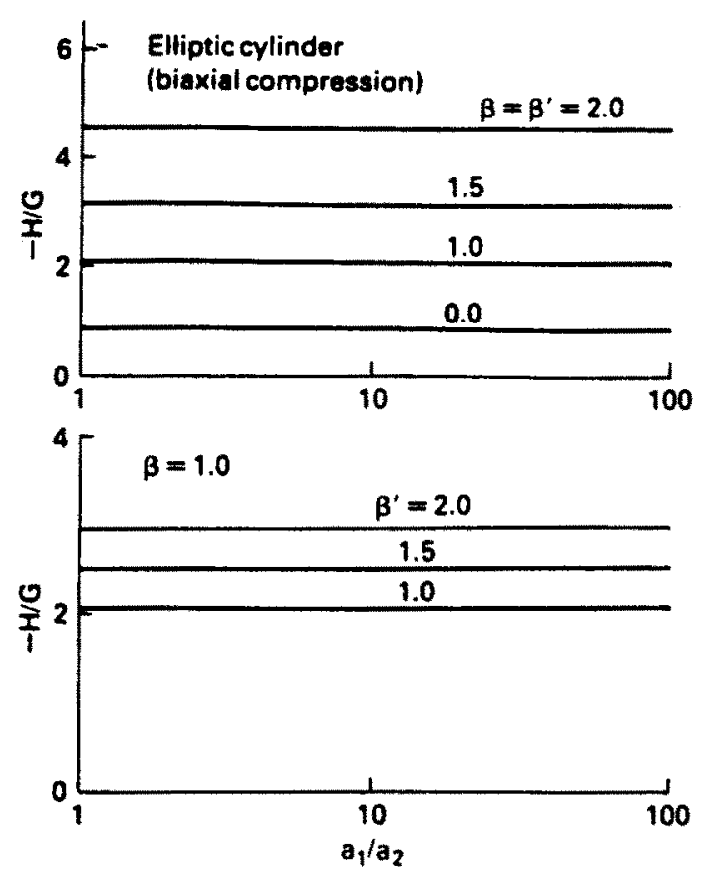

Fig. 9. Effect of axis ratio on critical $\boldsymbol{H}$ for elliptic cylinders in biaxial compression.

This means that strain-softening according to the Drucker-Prager plasticity model is stable against localization into a planar band even in an infinite space, provided that the plastic modulus magnitude does not exceed a certain limit.

By contrast, in the previous work (Bažant, 1988a), which considered only incrementally isotropic material properties, the value of the softening modulus $E_{t}$ (which is analogous to $H$ ) always approaches zero for an infinitely thick layer. This implies that the strain-softening cannot be stable in an infinitcly thick layer if the incremental modulus matrix is isotropic. Apparently, strain-softening of plasticity type (i.e. degradation of the yield limit) is not as destabilizing as some other forms of incremental material properties.
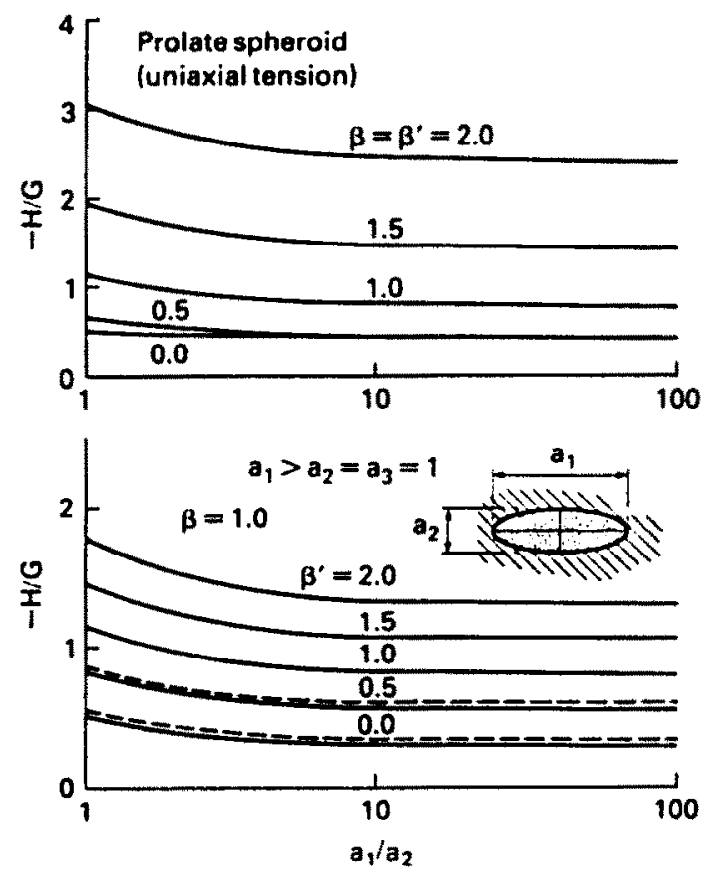

Fig. 10. Effect of axis ratio on critical $\boldsymbol{H}$ for prolate spheroids in uniaxial tension. 

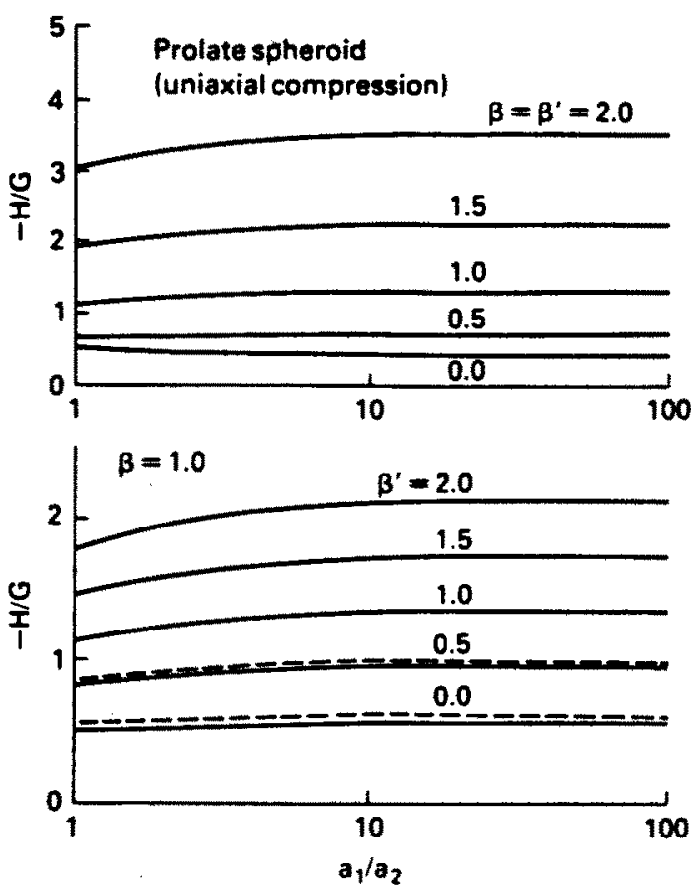

Fig. II. Erect of axis ratio on critical $\boldsymbol{H}$ for prolate spheroids in uniaxial compression.

Figures 6-9 show the dependence of the critical plastic modulus on the aspect ratio $a_{1} / a_{2}$ of a strain-softening ellipsoid degenerated into an inlinite elliptic cylinder, i.c. $a_{3} / a_{2} \rightarrow$ $\infty$. This limiting case is equivalent to localization in an infinite planar band within an infinite space. So the asymptotic values from Figs 6-9 and Figs 3-5 must coincide. They indeed do, which serves as a check on the correctness of the calculations.

Again it might have been expected that for an infinite aspect ratio $a_{1} / a_{2}$, the critical value of $\boldsymbol{H}$ should vanish. Surprisingly, it does not. The preceding comments apply here, too.

Furthermore, it should be noted that generally very high aspect ratios of the ellipsoid
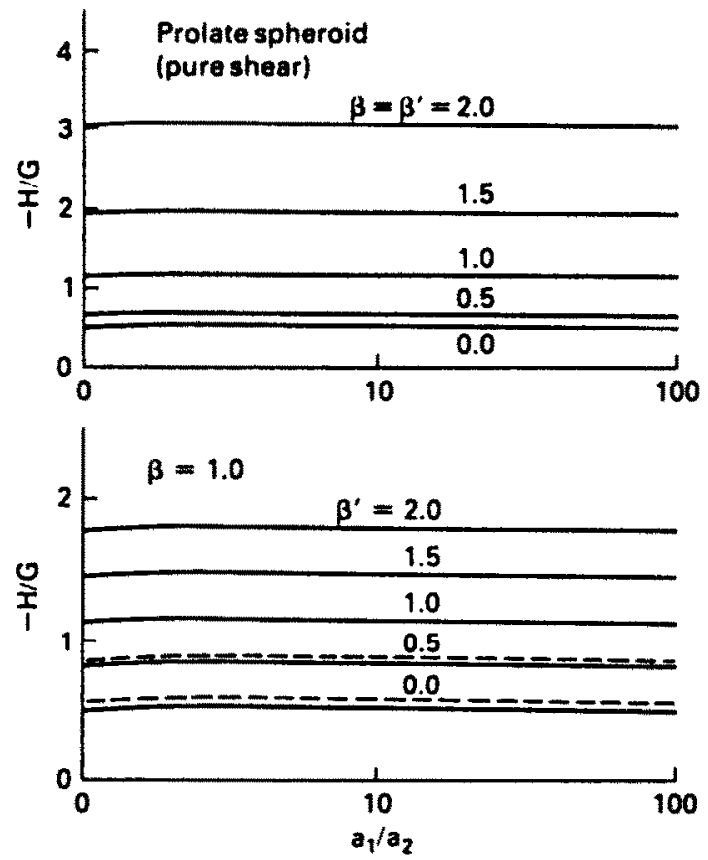

Fig. 12. Effect of axis ratio on critical $\boldsymbol{H}$ for prolate spheroids in pure shear. 

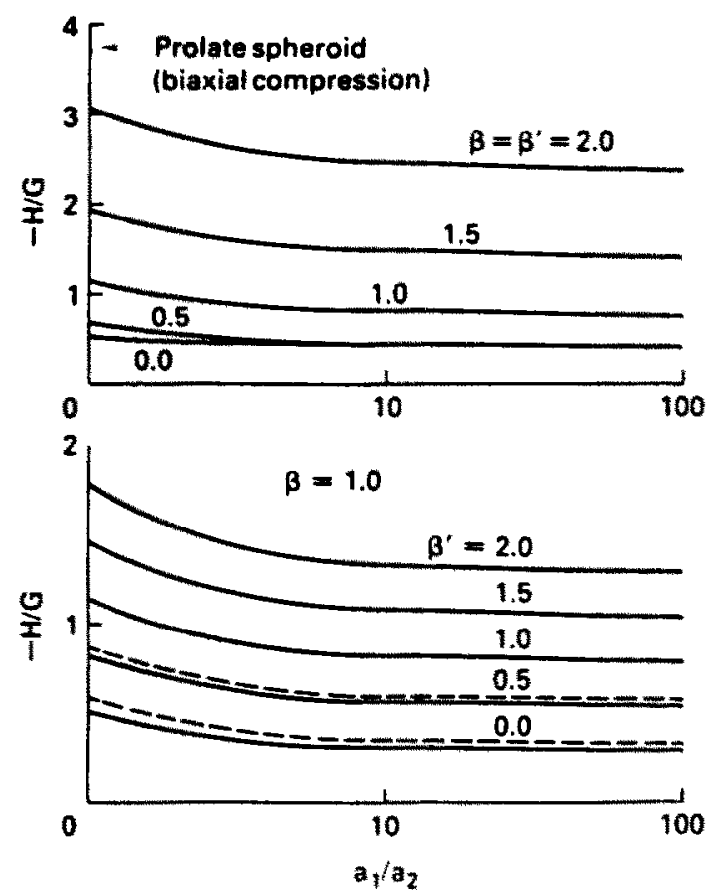

Fig. 13. Effect of axis ratio on critical $\boldsymbol{H}$ for prolate spheroids in biaxial compression.

are needed to reduce the critical magnitudes of $H$ near that for localization into a band in a layer. This of course means, similar to the conclusion of the previous study (Bažant. [988a, b), that the localization stability limits for a planar band are not well applicable to finite bodics representing typical structures.

Figures 10-13 show the dependence of the critical magnitudes of $H$ on the aspect ratio for prolate spheroids $\left(a_{2}=a_{3}\right)$. As the aspect ratio tends to infinity, the prolate spheroid approaches an infinite cylinder (a fiber). This is a different limiting case than before, and vanishing of the magnitude of $H$ is expected. It is also noteworthy that the strain-softening in prolate spheroids is much more stable than in elliptic cylinders, i.e. the critical magnitudes of $H$ are much larger in Figs 10-13 than in Figs 6-9.

The eigenvectors of matrix $Z$ or $Z_{i j}$ for the critical states for which the lowest eigenvalue is zero have been calculated too. For several typical cases, these eigenvectors are shown graphically in Fig. 14 for the volumetric (Rendulic) sections of the loading surface, and at bottom right also for the deviatoric cross-section (the values $v=0.18, \beta^{\prime}=1.0$ and uniaxial tension have been assumed in these calculations). In plotting these eigenvectors, it is understood that the axes of $\varepsilon^{v}$ and the deviatoric plastic strain intensity $\bar{\gamma}=\left(\frac{1}{2} e_{i j}^{p} e_{i j}^{p}\right)^{1 / 2}$ are superimposed on the axes of the volumetric stress and the deviatoric stress intensity. It is interesting that in the volumetric cross-section these eigenvectors are always inclined away from the normal to the loading function and toward the deviatoric axis, for all the dilatancy ratios $\beta$. These eigenvectors, of course, need not be normal to the loading function even if normality $\left(\beta^{\prime}=\beta\right)$ is assumed (it is the plastic strain incremental vector which must be normal if $\beta^{\prime}=\beta$ ).

\section{CONCLUSIONS}

Based on the general stability conditions for localization of strain-softening into planar bands and ellipsoidal domains, the critical values of the plastic modulus at stability limit are calculated for nonassociated and associated Drucker-Prager plasticity. Also presented is a direct derivation of the critical state conditions, which is simpler than the previous derivation of the stability conditions. It is found that strain-localization instabilities of the 

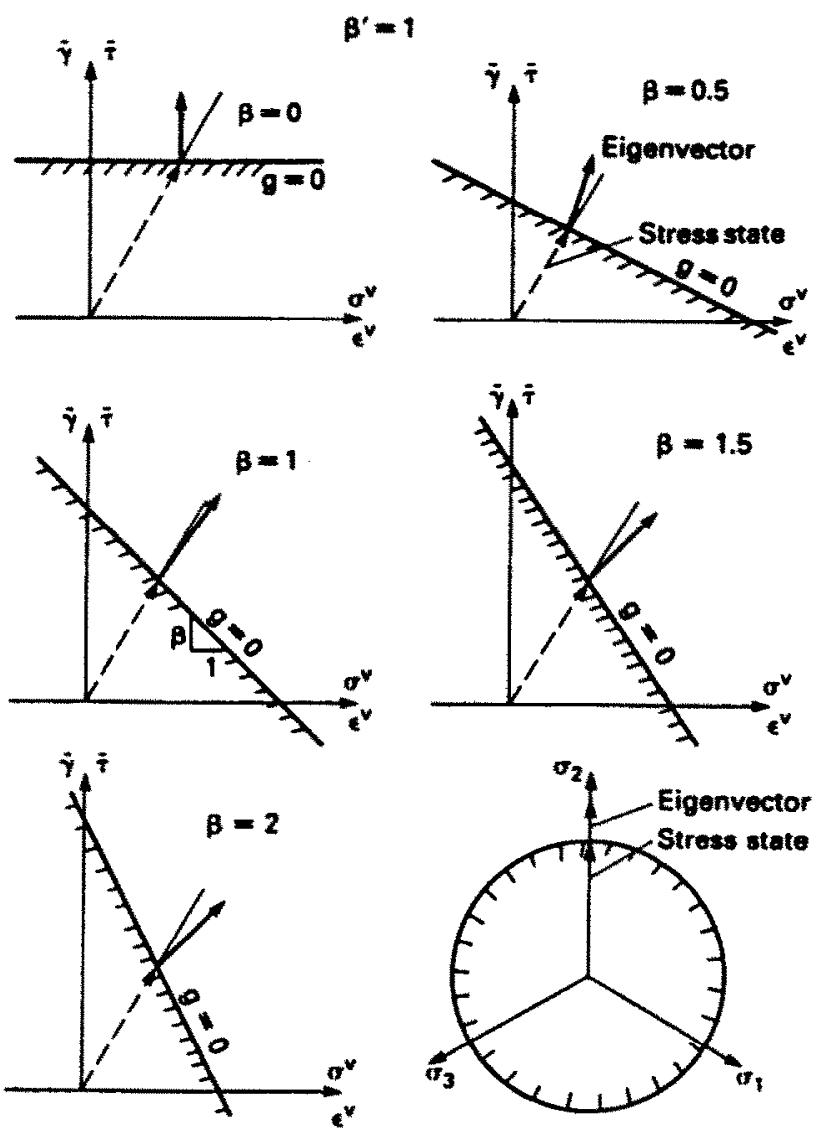

Fig. 14. Eigenvectors at the critical states in the volumetric and deviatoric sections.

type considered do not occur for a certain range of material parameters even if strainsoftening takes place. This is true also for strain-localization into a planar band in an infinite continuum, even though previous uniaxial analysis (Bažant. 1976) as well as the multiaxial analysis (Bažant, 1988a) indicated such situations to be always unstable for certain other constitutive models.

Acknowledgement-Partial financial support from the U.S. Air Force Oflice of Scientific Restarch under contract F49620-87.C-0030DEF with Northwestern University, monitored by Dr Spencer $T$. Wu, is gratefully acknowledged.

\section{REFERENCES}

Bazant, Z. P. (1976). Instability, ductility and size effect in strain-softening concrete. J. Engng Mech. Div.. ASCE 102 (EM2). 331-344; Discussions 103, 357-358, 775-777; 104, 501-502 (based on Struct. Engng Report No. 748/640. Northwestern University, August 1974).

Bafant, Z. P. (1982). Crack band model for fracture of geomaterials. Proc. 4 th Int. Conf. on Numerical Methods in Geomechanics, Edmonton. Canada (Edited by Z. Eisenstein), 3, 1137-1152.

Bułant, Z. P. (1986). Mechanics of distributed cracking. Appl. Mech. Revs, ASME 39 (5), 675-670.

Bajant, Z. P. (1987). Why continuum damage is nonlocal : justification by quasiperiodic microcrack array. Mech. Res. Communs $14(5 / 6), 407-419$.

Bajant, Z. P. (1988a). Softening instability: 1. Localization into a planar band. J. Appl. Mcch.. ASME 55, 517522.

Bazant, Z. P. (1988b). Softening instability: II. Localization into ellipsoidal regions. J. AppI. Mech., ASME 55, 523-529.

Bajant. Z. P. (1988c). Stable states and paths of structures with plasticity or damage. J. Engng Mach., ASCE $114,2013-2034$

Baiznt, Z. P. and Belytschko. T. B. (1987). Strain-softening continuum damage: localization and size effect. Preprints, Int. Conf, on Constitutive Laws for Engineering Materials, Tucson. AZ, Jan. 1987 (Edited by C. S. Desai et al.), Vol. 1, pp. 11-33. Elsevier, New York. 
Bażant, Z. P., Belytschko, T. B. and Chang. T. P. (1984). Continuum theory for strain-softening. J. Engng Mech. Div.. ASCE 110 (12). 1666-1692.

Bażant. Z. P. and Cedolin. L. (1979). Blunt crack band propagation in finite element analysis. J. Engng .Mech. Div.. ASCE 105(2). 297-315.

Bażant. Z. P. and Cedolin. L. (1980). Fracture mechanics of reinforced concrete. J. Engng Mech. Div.. ASCE 106 (EM6), 1287-1306: Discussion and Closure (1982) 108, 464-471.

Bažant. Z. P. and Lin. F. B. (1988). Nonlocal yield limit degradation. Int. J. Numer. Meth. in Engng 26, 18051823: see also Preprints, Int. Conf. On Computational Plasticity, Barcelona, April 1987 (Edited by E. Oñate, R. Owen and E. Hinton). 1757-1780. University of Wales, Swansea.

Bażant, Z. P. and Oh, B. H. (1983) - Crack band theory for fracture of concrete. Materials and Structures (RILEM, Paris) 16. 155-177.

Bażant. Z. P. and Pijaudier-Cabot. G. (1987). Modeling of distributed damage by nonlocal continuum with local strain. Preprints, 4 th Int. Conf. on Numerical Methods in Fracture Mechanics, San Antonio. Texas (Edited by A. P. Luxmore. R. J. Owen and M. F. Kanninen), 411-432.

Bażant. Z. P. and Pijaudier-Cabot. G. (1988). Nonlocal continuum damage, localization instability and convergence. J. Appl. Mech. ASME 55, 287-293.

Bażant. Z. P. and Pijaudier-Cabot. G. (1989). Measurement of characteristic length of nonlocal continuum. J. Enung . Vech. ASCE $115(4) .755-767$.

Chen. W. F. (1982). Plasticity in Reinforced Concrete. McGraw-Hill. New York.

Christensen. R. M. (1979). Mechanics of Compesite Materials. John Wiley, New York.

Darwin. D. (1985). Crack propagation in concrete - study of model parameters. In Proc. U.S.-Japan Seminar on Finite Element Analysis of Reinforced Concrete Structures. Tokyo (Edited by C. Meyer and H. Okamura), pp. 184-203. ASCE, New York.

de Borst. R. (1984). Application of advanced solution techniques to concrete cracking and non-associated plasticity. In Numerical Methods for Non-linear Problems (Edited by C. Taylor et al.). Vol. 2.314-332. Pineridge Press, Swansea, U.K.

de Borst. R. and Nauta, P. (1984). Smeared crack analysis of reinforced concrete beams and slabs failing in shear. In Proc. Int. Conf. on Computer-aided Analysis and Design of Concrete Structures, Split, Yugoslavia (Edited by F. Damjanic ef al. $)$, pp. 161-174. Pineridge Press, Swansca, U.K.

de Borst. R. and Nauta, P. (1985). Non-orthogonal cracks in a smeared finite clement model. Engng Comput. 2. 35.46.

Eshelby, J. D. (1957). The determination of the elastic field of an cllipsoidal inclusion and related problems. Proc. R. Snc. Lomd. A241, 376.

Fung, Y. C. (1965), Foundations of Solial Mtchanics. Prentice-Hall, Englewood Clifts, N.J.

IIill. R. (1962). Uniqueness criteria and extremum principles in self-adjoint problems of continuum mechanics. J. Mech. Phys. Solicts 10, 185 194.

Kinoshiti, N. and Mura, T. (1971). Elastic fields of inclusions in anisotropic media. Phys. Stat. Solidi (a) 5, 759 768 .

Lin. S. C. and Mura, T. (1973). Flastic tields of inclusions in anisotropic media (II). Phys. Stat. Solidi (a) 15. $281 \cdot 289$.

Mandel, J. (1966). Conditions de stabiliti et postulat de Drucker. In Rheology and Sosil Mechanics (Edited by 3. Kravtchenko and P. M. Sirieyes), pp. 58 68. Springer, Berlin.

Mróz. Z. (1966). On forms of constitutive laws for elastic -plastic solids. Arch. Mech. Stows. (Warszatw) 18, 3-35.

Mura, T. (1982). Micromechanics of Defects in Solids. Martinus Nijhoft, The Hague.

Owen, D. R. J. and Hinton. E. (1980). Finite Elements in Plasticity: Theory and Prattice. Pineridge Press, Swansea, U.K.

Pijaudier-Cabot, G. and Bažant, Z. P. (1987), Nonlocal damage: dynamic stability and convergence. Report No. 87-2/428n-1I. Center of Concrete and Geomaterials, Northwestern University, Evanston, IL. ; also Computers and Siructures 29(3), 503 507 (1988).

Rice, J. R. (1976). The localization of plastic deformation. Proc. I4th int. Congress of Theorerical and Applied Mechunics, Delft, The Netherlands, Vol. 1, 207-220.

Rudnicki, J. W. (1977). The inception of faulting in a rock mass with a weakened zone. J. Geophys. Res. 82(5). 844.854.

Rudnicki. J. W. and Rice, J. R. (1975). Conditions for the localization of deformation in pressure-sensitive dilattant matcrials. J. Mech. Phys. Solids 23, 371-394. 